\title{
The significance of plasma D-dimer level in predicting high risk factors of endometrial cancer
}

\author{
Yu-Jia Huang, Bi-Lan Li \\ Department of Gynecology, Shanghai First Maternity and Infant Hospital, Tongji University School of Medicine, Shanghai 201204, China \\ Contributions: (I) Conception and design: All authors; (II) Administrative support: All authors; (III) Provision of study materials or patients: All \\ authors; (IV) Collection and assembly of data: All authors; (V) Data analysis and interpretation: All authors; (VI) Manuscript writing: All authors; (VII) \\ Final approval of manuscript: All authors. \\ Correspondence to: Bi-Lan Li. Department of Gynecology, Shanghai First Maternity and Infant Hospital, Tongji University School of Medicine, \\ Shanghai 201204, China. Email: lorrain221@msn.com.
}

\begin{abstract}
Background: Activated clotting-fibrinolytic system is associated with poor outcome of cancer patients. This retrospective study aimed to evaluate the significance of plasma D-dimer level in predicting high risk factors of endometrial cancer (EC) patients.

Methods: Total 176 EC patients who underwent radiotherapy between January 2018 and June 2019 at Shanghai First Maternity and Infant Hospital were retrospectively analyzed. Their preoperative and postoperative plasma D-dimer levels were measured as routine assessment in our hospital, and analyzed for their association with clinicopathological data retrieved from medical records of the patients.

Results: High risk group had significantly higher 1 st day postoperative D-dimer levels. The 1 st day postoperative D-dimer predicted higher grade EC with the specificity of $63.7 \%$ and the sensitivity of $63.4 \%$; predicted late stage EC with the specificity of $83.7 \%$ and the sensitivity of $58.6 \%$; predicted deeper myoinvasion of EC with the specificity of $84.9 \%$ and the sensitivity of $43.3 \%$; predicted lymphovascular space invasion positive EC with the specificity of $84.0 \%$ and the sensitivity of $50.0 \%$; predicted lymph node metastasis of EC with the specificity of $50.9 \%$ and the sensitivity of $100 \%$; and predicted cervical invasion of EC with the specificity of $82.1 \%$ and the sensitivity of $65.0 \%$.
\end{abstract}

Conclusions: Increased postoperative plasma D-dimer levels accurately predicted high risk factors in patients with EC.

Keywords: Endometrial cancer (EC); D-dimer; risk factor; predict

Submitted Feb 23, 2020. Accepted for publication Apr 07, 2020.

doi: $10.21037 /$ tcr.2020.04.22

View this article at: http://dx.doi.org/10.21037/tcr.2020.04.22

\section{Introduction}

The cases of endometrial cancer (EC) diagnosed are increasing $(1,2)$. Surgery is still the standard treatment for early-stage EC (3). However, treatment strategies would be different for EC patients with different incidence of recurrence after surgery. Based on FIGO stage, grade and histotype, EC patients are divided into low risk and high risk groups for the risk of tumor recurrence (4). Biomarkers could help identify people with high risk of cancer (5). Therefore, it is urgent to develop new reliable biomarkers to stratify EC patients into risk categories for individualized therapy (6).

$\mathrm{D}$-dimer is involved in coagulation activation and has been applied for the diagnosis of venous thromboembolism (VTE) (7). In addition, D-dimer levels increase in several kinds of cancer, and correlate with poor prognosis and resistance to treatment (8). However, a recent study suggested that $\mathrm{D}$-dimer levels may not be a promising independent prognostic factor for EC (9). To further investigate the significance of plasma D-dimer in the prediction of high risk group of EC patients, in this 
retrospective study we analyzed the correlation of preoperative and postoperative plasma D-dimer levels with clinicopathological data of EC patients.

\section{Methods}

\section{Patients and procedures}

This retrospective study was approved by the Research Ethics Committee of Shanghai First Maternity and Infant Hospital. Demographic and clinical data were collected from medical records of $176 \mathrm{EC}$ patients who underwent radiotherapy between January 2018 and June 2019 at Shanghai First Maternity and Infant Hospital (Shanghai, China). The inclusion criteria included: EC diagnosis pathologically confirmed, no previous radiotherapy or chemotherapy, complete clinical data in medical record, and clinical staging was I-IV. The exclusion criteria included: thromboembolism in previous three months, using anticoagulation drugs, history of congenital thrombophilia, anticoagulant treatment, pregnancy, or stroke in previous six months, and cancer metastasis at the diagnosis. All procedures (total hysterosalpingo-oophorectomy and complete bilateral lymphadenectomy) were performed laparoscopically by the same experienced surgeon.

\section{Biochemical parameters}

After a 10-hour overnight fast, blood samples were collected from participants, and plasma was then analyzed using second-generation latex immunoassay kit for D-dimer on Sysmex CS-5100 (Siemens Diagnostics, Erlangen, Germany). The standard cut-off value of D-dimer in our hospital was $0.55 \mathrm{mg} / \mathrm{L}$. Platelet count levels were determined with an autoanalyzer (Sysmex XE-2100, Kobe, Japan).

\section{Diagnosis of deep vein thrombosis and pulmonary embolism}

An experienced ultrasonographist performed the compression ultrasonography according to standard procedures (Grey scale, B-mode, Color Doppler) with a high-end ultrasound scanner (Philips HD15 Ultrasound, USA) before and after surgery. If the patients had clinical signs of deep vein thrombosis, additional compression ultrasonography was performed. Pulmonary embolism was diagnosed based on computed tomography of the chest.
All Computed tomography pulmonary angiograms were analyzed by two experienced radiologists.

\section{Statistical analysis}

Data were analyzed by $t$-test, analysis of variance, MannWhitney U test, and Pearson's correlation analysis. Univariate and multivariate analyses based on Cox hazard model was used to determine prognostic factors. Receiver operating characteristic (ROC) curve was used to calculate cut-off value. Significance was indicated at $\mathrm{P}<0.05$.

\section{Results}

\section{Correlations between preoperative D-dimer plasma levels and clinicopathological parameters}

Total 176 EC patients were included and their clinicopathologic data were collected (Table 1). The average preoperative D-dimer level was $0.42 \mathrm{mg} / \mathrm{L}$ (range, $0.07-3.31 \mathrm{mg} / \mathrm{L}$ ), and the first day postoperative D-dimer level was $1.50 \mathrm{mg} / \mathrm{L}$ (range, $0.11-9.43 \mathrm{mg} / \mathrm{L}$ ). The preoperative plasma D-dimer level was below the normal level $(0.55 \mathrm{mg} / \mathrm{L})$ in $145 / 176(82.39 \%)$ patients and was above the normal level in $31 / 176(17.61 \%)$ patients. The median age was 54.6 years, and median body mass index (BMI) was $25.37 \mathrm{~kg} / \mathrm{m}^{2}$ for all patients. Higher D-dimer level showed significant correlation with older age $(\mathrm{P}=0.0067)$. There was no significant association of preoperative plasma $\mathrm{D}$-dimer level with platelet level $(\mathrm{P}=0.3846)$ or $\mathrm{BMI}(\mathrm{P}=0.9537)$.

\section{The first day postoperative D-dimer levels predicted tumor progression}

The associations between clinicopathologic characteristics and preoperative and postoperative plasma D-dimer levels were presented in Table 2. Compared to low-grade group, the first day postoperative $\mathrm{D}$-dimer levels were significantly higher in high-grade group $(\mathrm{P}=0.0135$; Figure $1 A)$. Compared to early-stage group, the first day postoperative $\mathrm{D}$-dimer levels were significantly higher in late-stage group $(\mathrm{P}<0.0001$; Figure $1 B)$. Compared to lymph node metastasis group, the first day postoperative $\mathrm{D}$-dimer levels were significantly higher in non-LN involvement group $(\mathrm{P}=0.0397$; Figure 1C). Compared to the $<1 / 2$ myoinvasion group, the first day postoperative $\mathrm{D}$-dimer levels were significantly higher in deep myoinvasion group $(\mathrm{P}=0.0003$; 
Table 1 Clinical-pathological features

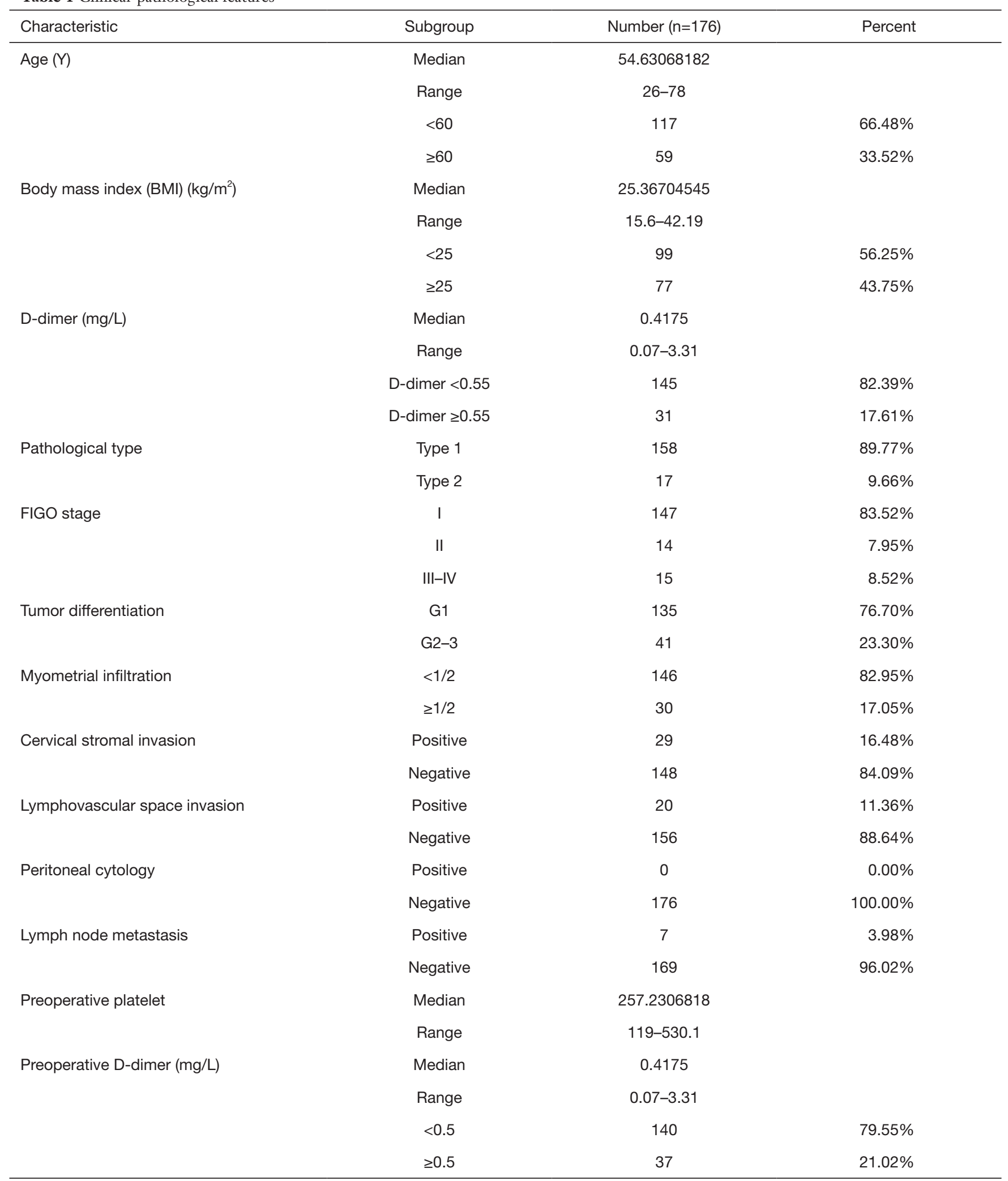

Table 1 (continued) 
Table 1 (continued)

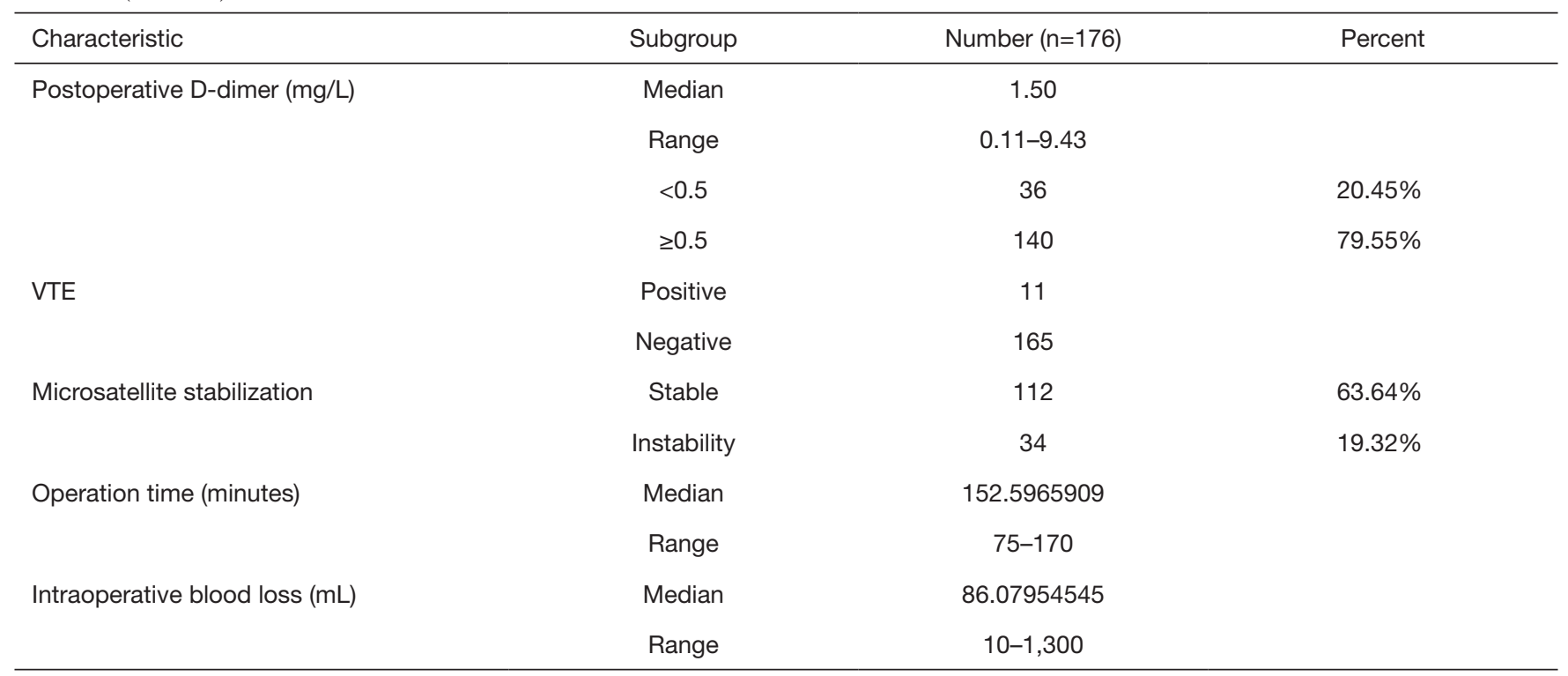

VTE, venous thromboembolism.

Figure 1D). Compared to lymphovascular space invasion negative group, the first day postoperative D-dimer levels were significantly higher in lymphovascular space invasion positive group ( $\mathrm{P}=0.0345$; Figure $1 E)$. Moreover, the 1st day postoperative $\mathrm{D}$-dimer level was significantly correlated with preoperative $\mathrm{D}$-dimer level $\left(\mathrm{R}^{2}=0.1795, \mathrm{P}<0.0001\right)$ (Figure 1F).

ROC curve analysis showed that optimal cut-off value for the 1st day postoperative D-dimer level was $1.25 \mathrm{mg} / \mathrm{L}$ for grade 2-3, $2.04 \mathrm{mg} / \mathrm{L}$ for stage III-IV, $2.205 \mathrm{mg} / \mathrm{L}$ for deep myoinvasion, $2.205 \mathrm{mg} / \mathrm{L}$ for lymphovascular space invasion, $1.07 \mathrm{mg} / \mathrm{L}$ for lymph node metastasis, and $2.04 \mathrm{mg} / \mathrm{L}$ for cervical invasion (Figure $2 A, B, C, D, E, F$ ). In addition, D-dimer predicted higher grade $\mathrm{EC}$ with the specificity of $63.7 \%$ and the sensitivity of $63.4 \%$ (AUC $0.662, \mathrm{P}=0.002)$; $\mathrm{D}$-dimer predicted late stage $\mathrm{EC}$ with the specificity of $83.7 \%$ and the sensitivity of $58.6 \%$ (AUC $0.754, \mathrm{P}<0.001)$; $\mathrm{D}$-dimer predicted deeper myoinvasion $(\geq 1 / 2$ myoinvasion) of EC with the specificity of $84.9 \%$ and the sensitivity of $43.3 \%$ (AUC $0.674, \mathrm{P}=0.003$ ); D-dimer predicted lymphovascular space invasion positive EC with the specificity of $84.0 \%$ and the sensitivity of $50.0 \%$ (AUC $0.683, \mathrm{P}=0.008$ ); $\mathrm{D}$-dimer predicted lymph node metastasis of EC with the specificity of $50.9 \%$ and the sensitivity of $100 \%$ (AUC 0.78, $\mathrm{P}=0.012$ ); D-dimer predicted cervical invasion of EC with the specificity of $82.1 \%$ and the sensitivity of $65.0 \%$ (AUC $0.742, \mathrm{P}=0.012$ ).

\section{Changes in preoperative and postoperative D-dimer levels were correlated with VTE events}

ROC curve showed significant association of D-dimer level with thrombus burden, and optimal cut-off values of D-dimer were 0.285 and $1.4 \mathrm{mg} / \mathrm{L}$ to predict VTE event. Notably, the preoperative D-dimer level was significantly affected by BMI, but not by the age and preoperative platelet. Preoperative D-dimer level was significantly correlated with 1 st postoperative day D-dimer level $(\mathrm{P}<0.0001$, Figure 3).

\section{Discussion}

EC most commonly presents with post-menopausal bleeding, and long-term bleeding may cause the abnormality of coagulation system. It is known that coagulation and fibrinolysis are activated in cancers, including gynecological cancers (10). In addition, VTE is a common complication in cancer patients and is listed as an important cause of cancer related death (11-13).

Plasma D-dimer is regarded as an important biomarker of fibrinolysis. The imbalance of the coagulation system is implicated in tumorigenesis $(14,15)$. It is proposed that cancer patients are in subclinical hypercoagulable condition, and fibrinolysis promotes tumor angiogenesis, leading to poor prognosis and even death. A recent study reported 
Table 2 Association between D-dimer levels and clinicopathological features in patients with endometrial cancer $(\mathrm{n}=176)$

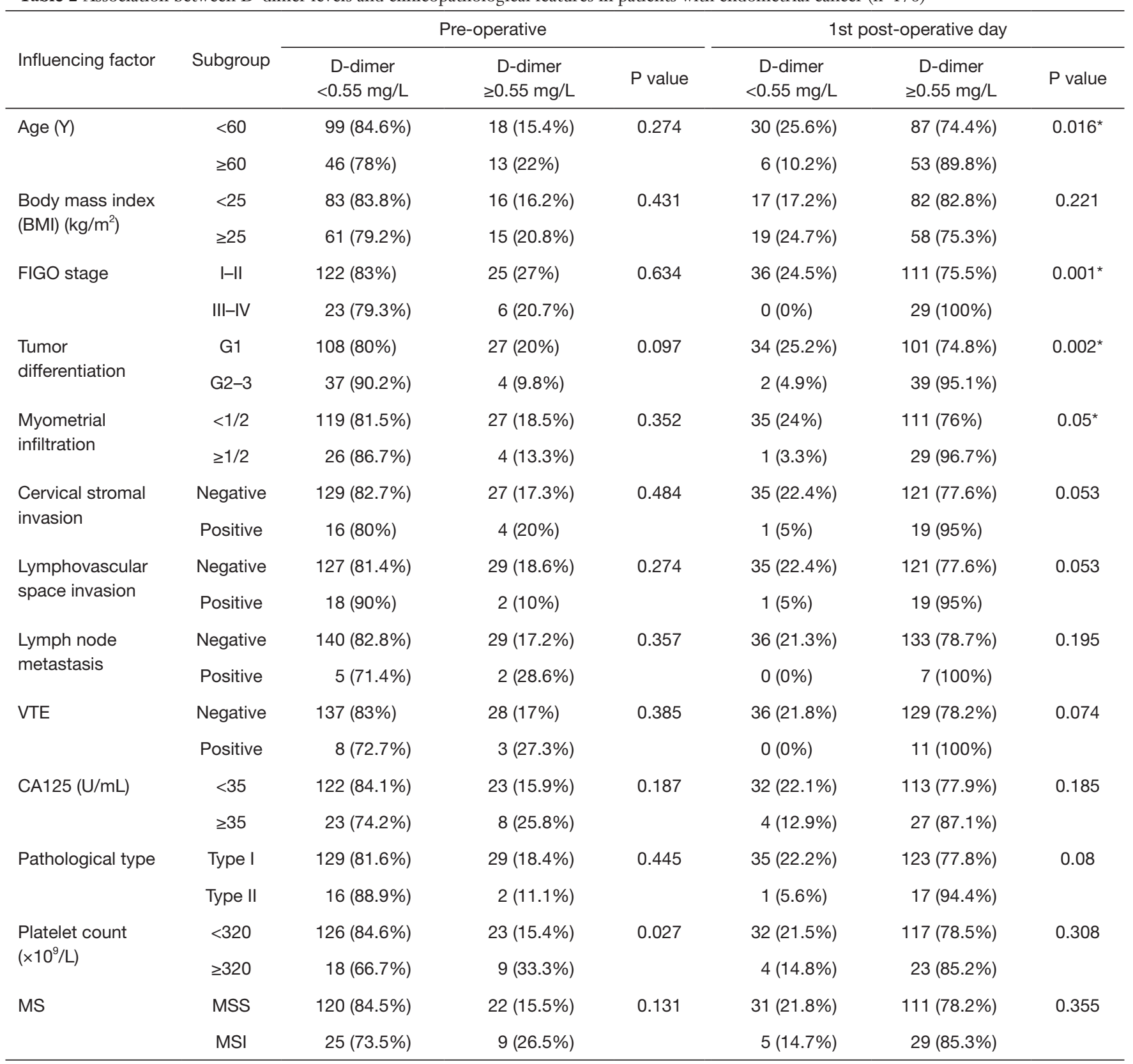

VTE, venous thromboembolism.

that D-dimer may predict postoperative recurrence and prognosis in patients with colorectal cancer metastasis. However, whether plasma D-dimer level could indicate postoperative recurrence and prognosis of EC patients remains unclear.

Currently, there are no valid noninvasive diagnostic methods available for EC. Biomarkers for EC would be invaluable for screening high-risk women, detecting primary and recurrent disease, and preoperative stratification of patients as high-risk and low-risk categories for personalized therapies (16).

Cancer-associated thrombosis could threaten the life of patients, and it is suggested that targeting the coagulation or fibrinolysis system could alleviate cancer progression (17). In most cancers, high D-dimer level usually indicated advanced cancer stage and increased risk 

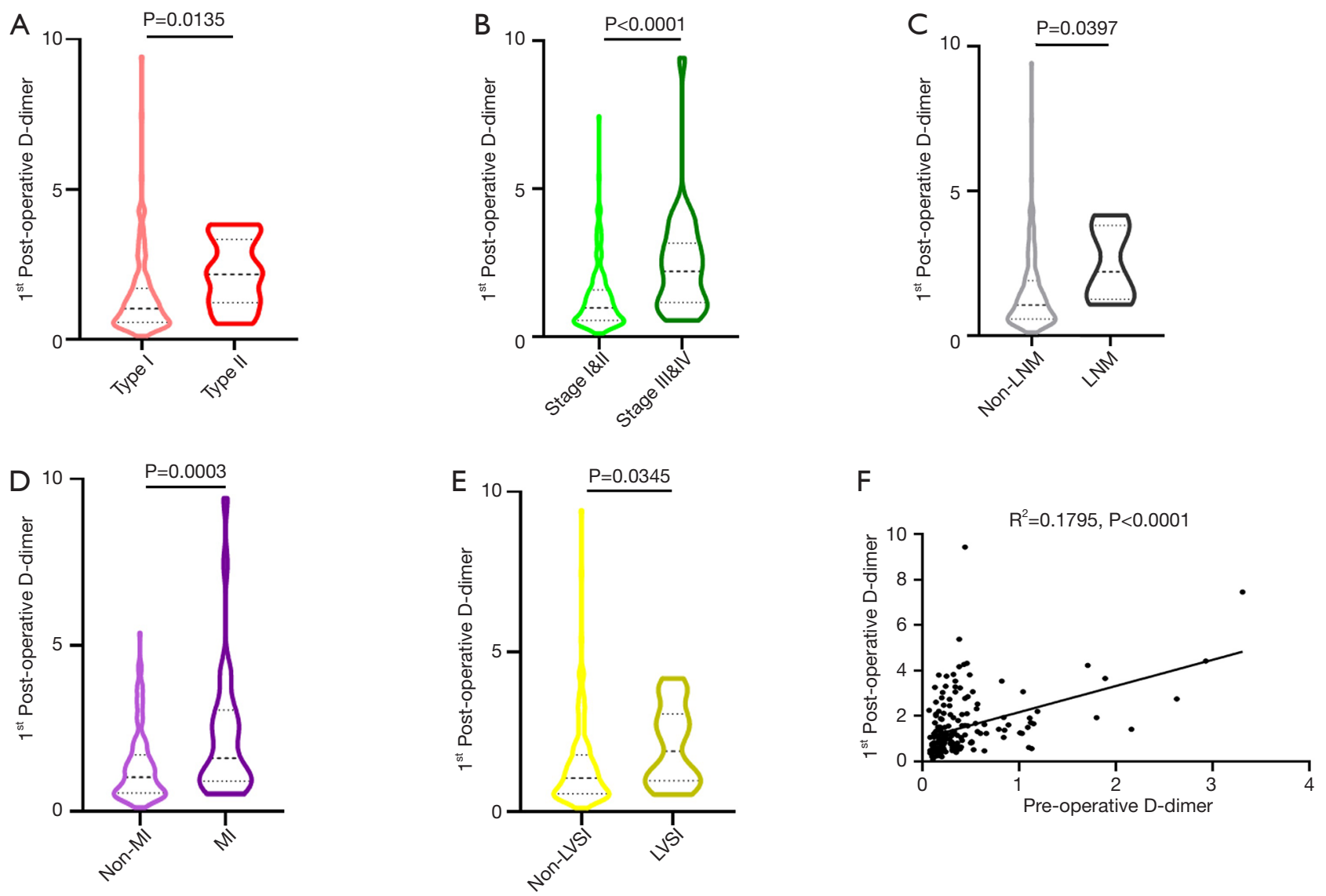

Figure 1 The association between 1st postoperative D-dimer level and clinicopathological parameters of EC patients. (A) Compared to low-grade group, high-grade group showed significantly higher the first day postoperative D-dimer levels $(\mathrm{P}=0.0135)$. (B) Compared to early-stage group, late-stage group showed significantly higher the first day postoperative $\mathrm{D}$-dimer levels $(\mathrm{P}<0.0001)$. (C) Compared to lymph node metastasis group, non-LN involvement group had significantly higher the first day postoperative $\mathrm{D}$-dimer levels $(\mathrm{P}=0.0397)$. (D) Compared to $<1 / 2$ myoinvasion group, the deep myoinvasion group had significantly higher the first day postoperative D-dimer levels ( $\mathrm{P}=0.0003)$. (E) Compared to lymphovascular space invasion negative group, lymphovascular space invasion positive group had significantly higher the first day postoperative $\mathrm{D}$-dimer levels $(\mathrm{P}=0.0345)$. (F) 1st day post-operative D-dimer showed significant correlation with preoperative D-dimer $(\mathrm{R} 2=0.1795, \mathrm{P}<0.0001)$. EC, endometrial cancer.

of death (18). High D-dimer level is associated with the progression and metastasis of gastric cancer, esophageal cancer, lung cancer, ovarian cancer, and nasopharyngeal carcinoma (19-22). Furthermore, plasma D-dimer level could evaluate the response to chemotherapy in colorectal cancer patients (23).

In present study, we analyzed the association of preoperative and postoperative plasma D-dimer levels with clinicopathological data of EC patients who had undergone surgery to treat EC. We found that the 1st day postoperative D-dimer level predicted higher grade EC with the specificity of $63.7 \%$ and the sensitivity of $63.4 \%$; predicted late stage EC with the specificity of $83.7 \%$ and the sensitivity of $58.6 \%$; predicted deeper myoinvasion of EC with the specificity of $84.9 \%$ and the sensitivity of $43.3 \%$; predicted lymphovascular space invasion positive EC with the specificity of $84.0 \%$ and the sensitivity of $50.0 \%$; predicted lymph node metastasis of EC with the specificity of $50.9 \%$ and the sensitivity of $100 \%$; and predicted cervical invasion of EC with the specificity of $82.1 \%$ and the sensitivity of $65.0 \%$. Our results suggest that 1 st day postoperative plasma D-dimer levels could predict high risk factors for EC recurrence such as higher grade, late stage, deeper myoinvasion, lymphovascular space invasion, lymph node metastasis and cervical invasion. More importantly, ROC analysis showed that postoperative 

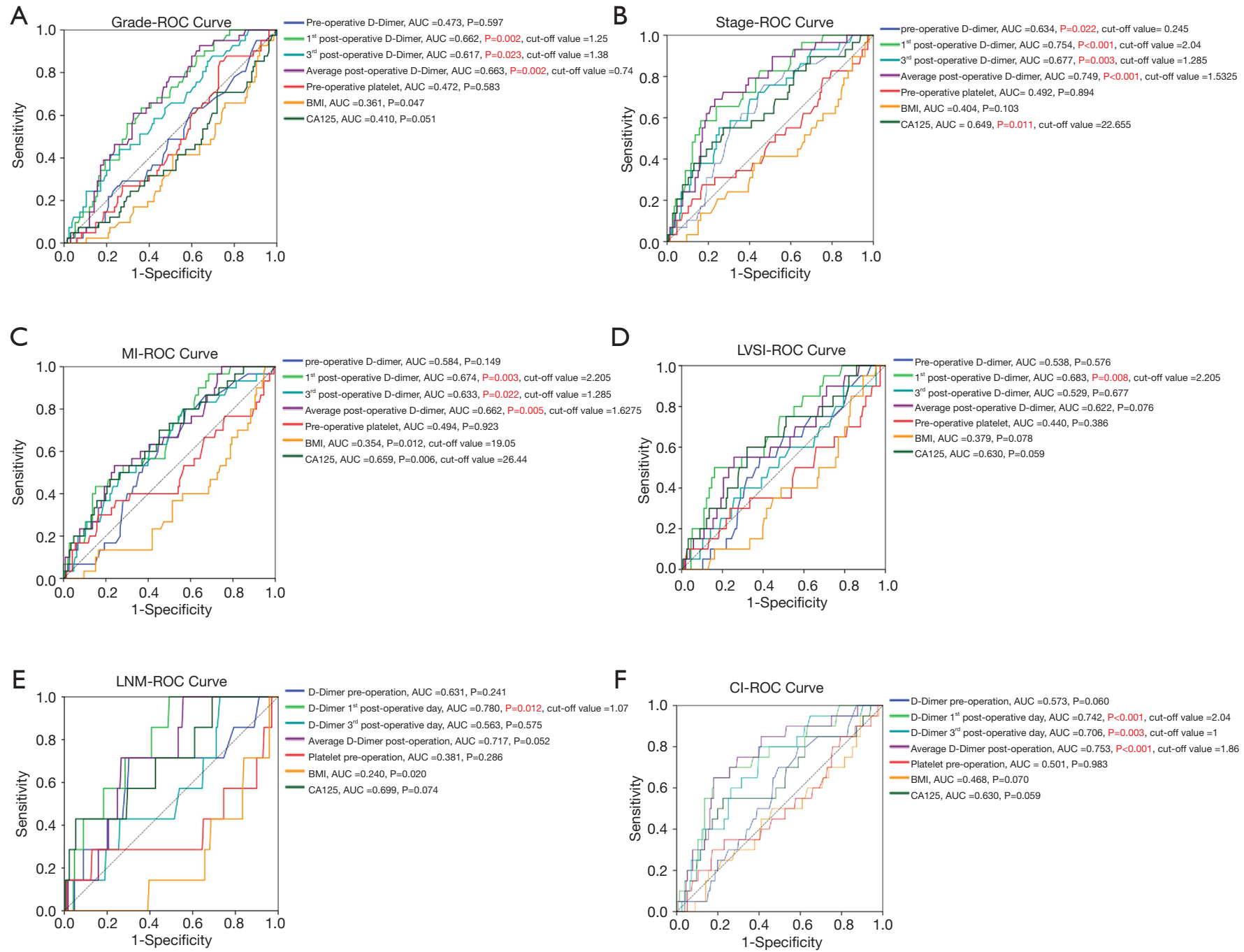

Figure 2 Optimized cut-off of the 1st day postoperative D-dimer levels for high risk of EC patients. (A) ROC curve for higher grade of EC showed that the optimal cut-off value for the 1 st postoperative $\mathrm{D}$-dimer was $1.25 \mathrm{mg} / \mathrm{L}(\mathrm{P}=0.002)$. (B) ROC curve for higher stage of EC showed that the optimal cut-off value for the 1 st postoperative $\mathrm{D}$-dimer was $2.04 \mathrm{mg} / \mathrm{L}(\mathrm{P}<0.001)$. (C) ROC curve for deep myoinvasion of EC showed that the optimal cut-off value for the 1 st postoperative $\mathrm{D}$-dimer was $2.205 \mathrm{mg} / \mathrm{L}(\mathrm{P}=0.003)$. (D) ROC curve for lymphovascular space invasion of EC showed that the optimal cut-off value for the 1st postoperative D-dimer was 2.205 mg/L (P=0.008). (E) ROC curve for lymph node metastasis of $\mathrm{EC}$ showed that the optimal cut-off value for the 1st postoperative $\mathrm{D}$-dimer was $1.07 \mathrm{mg} / \mathrm{L}(\mathrm{P}=0.012)$. (F) ROC curve for cervical metastasis of EC showed that the optimal cut-off value for the 1 st postoperative $\mathrm{D}$-dimer was $2.04 \mathrm{mg} / \mathrm{L}(\mathrm{P}<0.001)$. EC, endometrial cancer; ROC, receiver operating characteristic.

D-dimer level could be a better high-risk factor prediction than common tumor marker CA125 in EC patients. However, the accuracy of D-Dimer in the stratification of EC patients into risk categories need be confirmed in further large-scale and multi-center studies.

In conclusion, the activation of fibrinolysis system in cancer patients is an unfavorable clinical sign. As a degradation product of fibrin, increased postoperative plasma D-dimer level may be used to predict high risk of recurrence in $\mathrm{EC}$ patients.

\section{Acknowledgments}

Funding: This study was supported by grant from National 
A

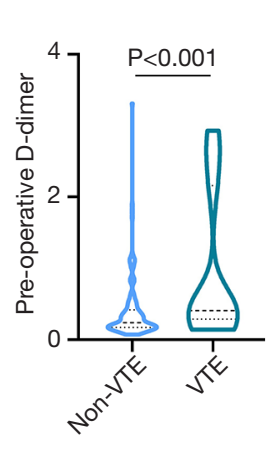

B

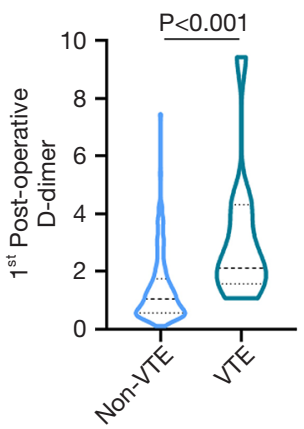

D

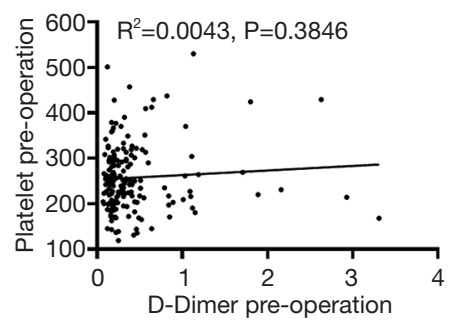

G

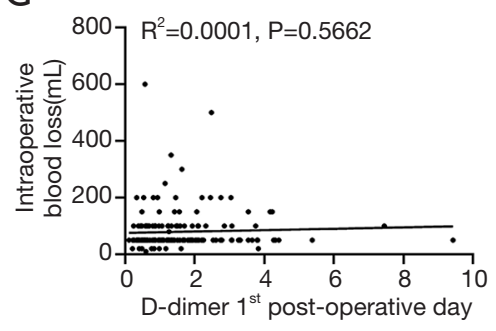

C $1.0 \quad$ VTE-ROC Curve

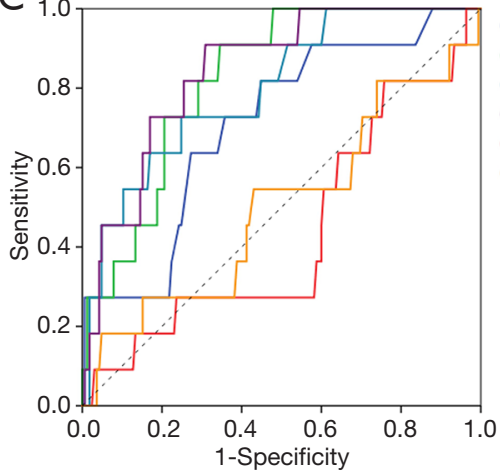

E

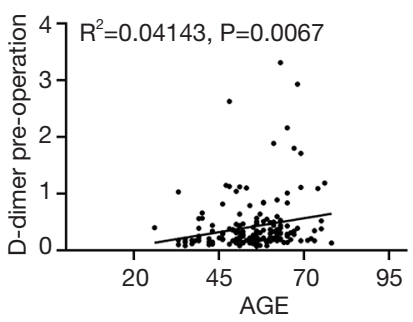

$\mathrm{H}$

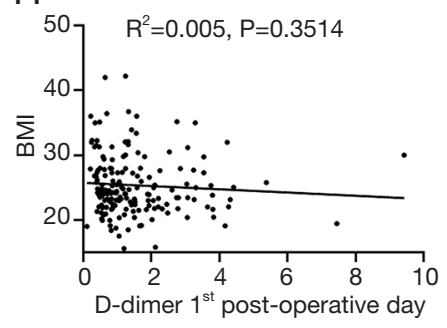

$\mathrm{F}$

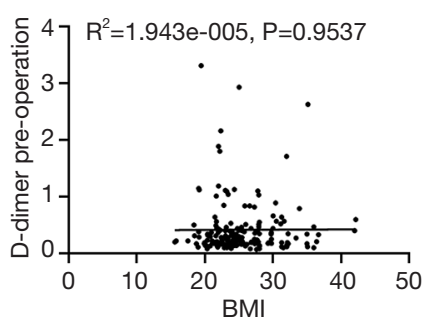

I

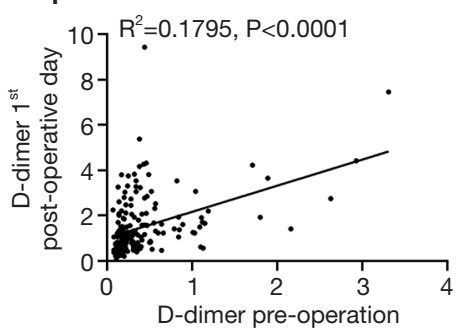

Figure 3 The relationship of preoperative and postoperative D-dimer levels with VTE events. (A) Compared to non-VTE patients, VTE patients showed significantly higher preoperative $\mathrm{D}$-dimer levels $(\mathrm{P}<0.001)$. (B) Compared to non-VTE patients, VTE patients showed significantly higher the first day postoperative $\mathrm{D}$-dimer levels $(\mathrm{P}<0.001)$. (C) Optimized cut-off was determined for preoperative and postoperative D-dimer levels using standard ROC curve analysis. (D,E,F) Preoperative D-dimer showed no correlation with preoperative platelet, body mass index and age. (G,H) 1st day postoperative D-dimer showed no correlation with preoperative body mass index and intraoperative blood loss. (I) 1 st day postoperative $\mathrm{D}$-dimer showed significant correlation with preoperative $\mathrm{D}$-dimer $\left(\mathrm{R}^{2}=0.1795\right.$, $\mathrm{P}<0.0001)$. VTE, venous thromboembolism; ROC, receiver operating characteristic.

Natural Science Foundation of China (No. 81602281).

\section{Footnote}

Provenance and Peer Review: This article was commissioned by the Guest Editor (Antonio Simone Laganà) for the series "Endometrial Cancer" published in Translational Cancer Research. The article was sent for external peer review organized by the Guest Editor and the editorial office.

Conflicts of Interest: Both authors have completed the
ICMJE uniform disclosure form (available at http://dx.doi. org/10.21037/tcr.2020.04.22). The series "Endometrial Cancer" was commissioned by the editorial office without any funding or sponsorship. The authors have no conflicts of interest to declare.

Ethical Statement: The authors are accountable for all aspects of the work in ensuring that questions related to the accuracy or integrity of any part of the work are appropriately investigated and resolved. The study was conducted in accordance with the Declaration of Helsinki 
(as revised in 2013). This retrospective study was approved by the Research Ethics Committee of Shanghai First Maternity and Infant Hospital. Individual informed consent was waived due to the retrospective nature of the study.

Open Access Statement: This is an Open Access article distributed in accordance with the Creative Commons Attribution-NonCommercial-NoDerivs 4.0 International License (CC BY-NC-ND 4.0), which permits the noncommercial replication and distribution of the article with the strict proviso that no changes or edits are made and the original work is properly cited (including links to both the formal publication through the relevant DOI and the license). See: https://creativecommons.org/licenses/by-nc-nd/4.0/.

\section{References}

1. Siegel RL, Miller KD, Jemal A. Cancer statistics, 2018. CA Cancer J Clin 2018;68:7-30.

2. Chen $\mathrm{W}$, Zheng R, Baade PD, et al. Cancer statistics in China, 2015. CA Cancer J Clin 2016;66:115-32.

3. Morice P, Leary A, Creutzberg C, et al. Endometrial cancer. Lancet 2016;387:1094-108.

4. Salvesen HB, Haldorsen IS, Trovik J. Markers for individualised therapy in endometrial carcinoma. Lancet Oncol 2012;13:e353-61.

5. Busch EL, Crous-Bou M, Prescott J, et al. Adiponectin, Leptin, and Insulin-Pathway Receptors as Endometrial Cancer Subtyping Markers. Horm Cancer 2018;9:33-9.

6. Weinberger V, Bednarikova $M$, Hausnerova J, et al. A Novel Approach to Preoperative Risk Stratification in Endometrial Cancer: The Added Value of Immunohistochemical Markers. Front Oncol 2019;9:265.

7. Cai H, Ye X, Zheng $W$, et al. Pitfalls in the diagnosis and initial management of acute cerebral venous thrombosis. Rev Cardiovasc Med 2018;19:129-33.

8. Prandoni P, Noventa F, Ghirarduzzi A, et al. The risk of recurrent venous thromboembolism after discontinuing anticoagulation in patients with acute proximal deep vein thrombosis or pulmonary embolism. A prospective cohort study in 1,626 patients. Haematologica 2007;92:199-205.

9. Li Q, Cong R, Kong F, et al. Fibrinogen Is A Coagulation Marker Associated With The Prognosis Of Endometrial Cancer. Onco Targets Ther 2019;12:9947-56.

10. Ay C, Dunkler D, Pirker R, et al. High D-dimer levels are associated with poor prognosis in cancer patients. Haematologica 2012;97:1158-64.

11. Lyman GH, Khorana AA. Cancer, clots and consensus: new understanding of an old problem. J Clin Oncol 2009;27:4821-6.

12. Adam SS, Key NS, Greenberg CS. D-dimer antigen: current concepts and future prospects. Blood 2009;113:2878-87.

13. Ye $\mathrm{S}$, Zhang $\mathrm{W}$, Yang J, et al. Pattern of Venous Thromboembolism Occurrence in Gynecologic Malignancy: Incidence, Timing, and Distribution a 10Year Retrospective Single-institutional Study. Medicine (Baltimore) 2015;94:e2316.

14. Kuderer NM, Ortel TL, Francis CW. Impact of venous thromboembolism and anticoagulation on cancer and cancer survival. J Clin Oncol 2009;27:4902-11.

15. Falanga A, Marchetti M, Vignoli A. Coagulation and cancer: biological and clinical aspects. J Thromb Haemost 2013;11:223-33.

16. Rizner TL. Discovery of biomarkers for endometrial cancer: current status and prospects. Expert Rev Mol Diagn 2016;16:1315-36.

17. Korte W. Changes of the coagulation and fibrinolysis system in malignancy: their possible impact on future diagnostic and therapeutic procedures. Clin Chem Lab Med 2000;38:679-92.

18. Ma X, Li Y, Zhang J, et al. Prognostic role of D-dimer in patients with lung cancer: a meta-analysis. Tumour Biol 2014;35:2103-9.

19. Liu L, Zhang X, Yan B, et al. Elevated plasma D-dimer levels correlate with long term survival of gastric cancer patients. PLoS One 2014;9:e90547.

20. Liu P, Wang Y, Tong L, et al. Elevated preoperative plasma $\mathrm{D}$-dimer level is a useful predictor of chemoresistance and poor disease outcome for serous ovarian cancer patients. Cancer Chemother Pharmacol 2015;76:1163-71.

21. Feng JF, Yang X, Chen S, et al. Prognostic Value of Plasma D-dimer in Patients with Resectable Esophageal Squamous Cell Carcinoma in China. J Cancer 2016;7:1663-7.

22. Chen WH, Tang LQ, Wang FW, et al. Elevated levels of plasma D-dimer predict a worse outcome in patients with nasopharyngeal carcinoma. BMC Cancer 2014;14:583.

23. Inanc M, Er O, Karaca H, et al. D-dimer is a marker of response to chemotherapy in patients with metastatic colorectal cancer. J BUON 2013;18:391-7.

Cite this article as: Huang $\mathrm{YJ}, \mathrm{Li} \mathrm{BL}$. The significance of plasma D-dimer level in predicting high risk factors of endometrial cancer. Transl Cancer Res 2020;9(12):7688-7696. doi: $10.21037 /$ tcr.2020.04.22 